\title{
\&VIII SIBRAGEC

\section{ANÁLISE DO RISCO RELACIONADO À FALTA DE TERMINALIDADE DE SERVIÇOS EM UMA OBRA RESIDENCIAL ${ }^{1}$}

\author{
LIMA, Josué Nazário (1); ALMEIDA, Gabriel Gouveia (2); ULHÔA, Cécile \\ Guimarães (3); BRANDSTETTER, Maria Carolina Gomes de Oliveira (4) \\ (1) Universidade Federal de Goiás, josuenazario@discente.ufg.br \\ (2) Universidade Federal de Goiás, gabrielgouveia261@gmail.com \\ (3) Universidade Federal de Goiás, cecileulhoa@uol.com.br \\ (4) Universidade Federal de Goiás, maria_carolina_brandstetter@ufg.br
}

\begin{abstract}
RESUMO
A ausência de terminalidade das etapas de um empreendimento pode gerar atraso nos serviços subsequentes e transtornos na gestão da obra. Apesar de sua relevância, há uma lacuna de pesquisas que buscam estudar o risco associado a serviços não conclusos em obras. É este o contexto deste trabalho. Buscou-se estudar os riscos relacionados à falta de terminalidade de serviços e propor mecanismos para correção e melhoria nos processos produtivos. Um estudo de caso foi conduzido em uma obra de múltiplos pavimentos em fase de acabamento na cidade de Goiânia. A coleta de dados foi realizada em visitas com acompanhamento da administração. A análise de risco permitiu a identificação da causa raiz de 236 ocorrências, as quais foram agrupadas em 14 categorias de acordo com a origem da falta de terminalidade. Para a criação do índice de risco, considerou-se simultaneamente a frequência em que ocorre uma falta de terminalidade e o impacto causado na obra em relação a aditivos contratuais. A identificação da causa raiz levou a uma classificação em macrocategorias relacionadas às diferentes origens dos problemas, como projeto, qualidade, controle, comunicação, entre outros. Os resultados apontam o maior risco associado às origens relacionadas aos projetos e à gestão da qualidade.
\end{abstract}

Palavras-chave: Terminalidade, Riscos, Atrasos de serviços, Planejamento.

\begin{abstract}
The lack of finish of the summary tasks of an enterprise may cause delay in subsequent services and nuisances in the management of the construction site. Despite its relevance, there is a gap in research that seeks to study the risk associated with services not completed in a construction site. This is the content of this work. We sought to study the risks related to the lack of conclusion of tasks and to propose mechanisms for correction and improvement in production processes. A case study was carried out on a multi-storey building being completed in the city of Goiania. Data collection was carried out during visits monitored by the management team. The risk analysis allowed the identification of the root cause of 236 occurrences, which were grouped into 14 categories according to the origin of the lack of finish. For the creation of the risk index, it was considered both the frequency in which a lack of finish occurs and the impact caused on the construction site in relation to contractual additives. The identification of the root cause led to a classification in macro-categories related to the different origins of the problems, such as drawings, quality, control, communication, among others. The results indicate the greatest risk associated with origins related to projects and quality management.
\end{abstract}

Keywords: Finish, Risk, Delay in services, Planning

\footnotetext{
1 LIMA, J. N. de; ALMEIDA, G. G. de; ULHÔA, C. G.; BRANDSTETTER, M. C. G. de O. Análise do risco relacionado à falta de terminalidade de serviços em uma obra residencial. In: SIMPÓSIO BRASILEIRO DE GESTÃO E ECONOMIA DA CONSTRUÇÃO, 12., 2021, Maceió, Alagoas. Anais[...] Porto Alegre: ANTAC, 2021. p.1-8. Disponível em: https://eventos.antac.org.br/index.php/sibragec/article/view/639. Acesso em: 2 out. 2021.
} 


\section{INTRODUÇÃO}

Com o intuito de alcançar eficiência, toda administração preocupa-se com custos e seus fatores associados, tais como o gerenciamento da equipe e dos empreiteiros, desperdícios, retrabalhos, falta de terminalidade dos serviços, além de problemas relacionados à qualidade na compra de materiais, equipamentos e contratações de serviços (ULHÔA, 2012; RENUKA; KAMAL; UMARANI, 2017; AKHUND et al., 2018).

Ulhôa (2017) afirma que experiências com empreendimentos entregues mostram que inúmeros serviços são dados como concluídos, mesmo que haja falhas na qualidade ou estejam incompletos. Mediante tal situação, empresas visam intensificar o acompanhamento das obras, melhorando o controle a fim de evitar os retrabalhos e falta de terminalidade dos serviços.

Esta pode ser estabelecida como o parâmetro para que um serviço, em um espaço prédefinido como um apartamento ou pavimento, possa ser dado como finalizado sem que haja a necessidade de retorno para conclusão, ou seja, sem que haja resquícios do mesmo. Em uma obra, esses critérios são avaliados para que atividades com predecessoras definidas possam ser iniciadas no tempo planejado (ULHÔA, 2012).

A ausência de terminalidade implica na maioria dos casos em atraso nos serviços subsequentes gerando inúmeros transtornos na gestão da obra. Um serviço não terminado demandará mais mão de obra, custos e tempo, fazendo-se necessário um controle mais preciso da evolução dos serviços, a fim de apontar os possíveis erros no processo e reparálos de forma rápida.

Ainda, as empresas estão sujeitas a enfrentar inúmeras influências que podem surgir de fatores internos e externos tornando incerto o alcance dos seus objetivos. Esse efeito é denominado risco de acordo com o estabelecido na ABNT NBR ISO 31000:2018. Um evento como um desvio de prazo, em decorrência da falta de terminalidade dos serviços, pode ser definido como um evento de risco, devido ao efeito da incerteza quanto ao alcance do prazo programado.

Conforme estabelece a norma ISO/IEC 31010:2012, vários métodos podem ser utilizados para identificação dos impactos e probabilidades das fontes e eventos de risco. A escolha do método depende da disponibilidade de dados confiáveis, porém quanto maior a precisão na definição do impacto e da probabilidade para cada fator ou evento de risco, mais assertiva será a tomada de decisão acerca de ações futuras sobre tratamento dos riscos (BRANDSTETTER; RIBEIRO, 2020).

O tema da terminalidade dos serviços pode ser visto no contexto de estudos relacionados ao conceito de antecipações gerenciais no planejamento da produção (MACHADO, 2003; SANTOS, 2004) e ao conceito de making-do, que se destaca por representar uma fonte de outras perdas como o retrabalho ou problemas relativos à qualidade (SOMMER, 2010; FORMOSO et al., 2011; FORMOSO et al., 2017; FIREMAN et al., 2013; SANTOS; SANTOS, 2017).

Diante do contexto exposto, a proposta do presente trabalho consiste em analisar o risco relacionado à falta de terminalidade de serviços em uma obra residencial. Objetiva-se apresentar uma proposta de avaliação do risco da falta de terminalidade de serviços de modo a subsidiar mecanismos para correção e mitigação de futuros problemas similares.

\section{MÉTODO DE PESQUISA}

Segundo o que preconiza a ABNT NBR ISO 31010: 2012, o nível de risco é determinado a partir da combinação de consequências ou impactos e suas probabilidades ou frequências para eventos identificados de risco. Para atingir o objetivo do trabalho, 
buscou-se o desenvolvimento de um método que permitisse a identificação das principais ocorrências, a quantificação da frequência e o impacto destas, para o desenvolvimento de um índice de risco em relação à falta de terminalidade dos serviços.

A categorização para as falhas de terminalidade foi proposta pelos autores, desenvolvida a partir das observações in loco e entrevistas não estruturadas com o corpo gerencial da obra.

A escolha de uma empresa para o desenvolvimento da proposta considerou os critérios: experiência e consolidação no mercado, interesse em participar e contribuir com trabalhos acadêmicos, além de possuir um Sistema de Gestão Integrado.

Posteriormente definiu-se a obra em que o estudo de caso foi realizado. A mesma teve início em agosto/2018 com previsão de término para abril/2021, o que possibilitou a coleta de dados para a pesquisa, incluindo os dados pré-existentes de avanço físico dos meses anteriores ao início da pesquisa.

O empreendimento é composto por duas edificações de múltiplos pavimentos com 96 unidades habitacionais.

Foram disponibilizados os dados relacionados ao avanço físico, indicadores físicos, registros fotográficos e documentação de reuniões mensais de produção. As visitas técnicas para coleta de evidências foram realizadas no segundo semestre do ano de 2020, sob a supervisão da engenheira responsável. Para o estudo optou-se pela escolha de serviços de acabamentos como revestimento de parede, forro de gesso, piso cerâmico, pintura, esquadrias de madeira e instalação de louças.

Inicialmente, foram pontuadas as principais faltas de terminalidade observadas nos apartamentos visitados. Estas foram relacionadas numa planilha, conforme ilustrado na Figura 1, para registrar quais serviços apresentavam pendências e em quais unidades habitacionais. Além dos dados coletados na planilha, também foram realizados registros fotográficos das ocorrências detectadas. É importante ressaltar que foram consideradas faltas de terminalidade serviços inacabados não planejados. Cerca de $70 \%$ das unidades residenciais foram avaliadas.

Figura 1 - Parte da planilha utilizada para a coleta e quantificação dos dados.

\begin{tabular}{|c|c|c|c|c|c|c|c|c|c|c|c|c|c|c|c|c|c|}
\hline \multirow[b]{2}{*}{ Ocorrência } & \multirow[b]{2}{*}{ Terminalidade } & \multicolumn{15}{|c|}{ APARTAMENTOS } & \multirow[b]{2}{*}{ Macrocategoria } \\
\hline & & 101 & 102 & 201 & 203 & 301 & 302 & 304 & 401 & 402 & 403 & 1104 & 1201 & 1203 & 1301 & 1303 & \\
\hline 1 & $\begin{array}{l}\text { Descrição da } \\
\text { ocorrência }\end{array}$ & & $\mathrm{x}$ & & $\mathrm{x}$ & & $\mathrm{x}$ & & & $\mathrm{x}$ & $\mathrm{x}$ & & & $\mathrm{x}$ & & $\mathrm{x}$ & Descrição \\
\hline
\end{tabular}

Fonte: Autoria própria (2021)

Após coleta e com o intuito de identificar a causa raiz de cada ocorrência de falta de terminalidade, foi realizada a análise dos dados a partir da ferramenta dos cinco porquês com o apoio da equipe gerencial da obra.

A identificação da causa raiz levou a uma classificação em 6 macrocategorias relacionadas às diferentes origens dos problemas, as quais foram adaptadas do trabalho de Ulhôa (2017): falha na análise de projetos, falha no controle, falha na gestão de qualidade, incompatibilização de projetos, falha na comunicação e falha de projetos (referente à solução técnica do projeto).

Posteriormente as ocorrências foram quantificadas em termos de frequência (número de ocorrências detectadas na amostra coletada) e depois analisadas quanto ao seu impacto. Para a análise de frequência foram criados critérios conforme Quadro 1. 
Quadro 1 - Categorização da frequência das ocorrências

\begin{tabular}{|c|c|c|}
\hline Índice & Frequência & Critérios \\
\hline 1 & Baixa & De 0,0 a $3,0 \%$ em relação ao total de ocorrências \\
\hline 2 & Média & De 3,1 a $9,0 \%$ em relação ao total de ocorrências \\
\hline 3 & Alta & Maior ou igual a $9,1 \%$ em relação ao total de ocorrências \\
\hline
\end{tabular}

Fonte: Autoria própria (2021)

Para a análise do impacto foram propostos os critérios apresentados no Quadro 2.

Quadro 2 - Categorização do impacto das ocorrências

\begin{tabular}{|c|c|l|}
\hline Nível & Impacto & \multicolumn{1}{c|}{ Critérios } \\
\hline 1 & Baixo & $\begin{array}{l}\text { Não houve impacto na sequência natural dos serviços e nem na contratação de } \\
\text { mão de obra, porém houve aditivos no custo. }\end{array}$ \\
\hline 2 & Médio & $\begin{array}{l}\text { Houve impacto na sequência natural ou na contratação de mão de obra e houve } \\
\text { aditivos no custo. }\end{array}$ \\
\hline 3 & Alto & $\begin{array}{l}\text { Houve impacto na sequência natural, na contratação de mão de obra e aditivos } \\
\text { no custo. }\end{array}$ \\
\hline
\end{tabular}

Fonte: Autoria própria (2021)

Os três níveis buscaram representar de forma resumida a severidade das ocorrências. No nível 1 apenas o custo foi impactado, ou seja, as atividades subsequentes seguiram normalmente e não se fez necessária a contratação de uma nova mão de obra para corrigir a falta de terminalidade. O nível 2 representa ocorrências que, além do custo extra, ou geraram impacto nos serviços subsequentes ou a necessidade de contratação de mão de obra não programada. $\mathrm{O}$ nível 3 agrupa as ocorrências que impactaram de forma mais severa, no custo, nos serviços subsequentes e na necessidade de mão de obra extra.

Para o índice de risco, considerou-se simultaneamente a frequência em que ocorre uma falta de terminalidade e o impacto causado por ela na obra. Este índice é resultado da multiplicação da frequência e impacto e o Quadro 3 ilustra a sua classificação.

Quadro 3 - Índice de risco da falta de terminalidade

\begin{tabular}{|c|c|l|}
\hline $\begin{array}{c}\text { Índice de } \\
\text { Risco }\end{array}$ & $\begin{array}{c}\text { Intervalo de } \\
\text { Valores }\end{array}$ & \multicolumn{1}{c|}{ Grau de urgência das intervenções } \\
\hline Baixo & $\mathrm{IR} \leq 2$ & Requer intervenções para melhoria, porém sem caráter de urgência \\
\hline Moderado & $2<\mathrm{IR}<6$ & $\begin{array}{l}\text { Requer ações corretivas visando diminuir a probabilidade de ocorrência } \\
\text { em empreendimentos futuros }\end{array}$ \\
\hline Elevado & $\mathrm{IR} \geq 6$ & $\begin{array}{l}\text { Requer ações corretivas com análise critica das etapas que se relacionam } \\
\text { com o serviço e controle, para que as ocorrências não se repitam em } \\
\text { empreendimentos futuros }\end{array}$ \\
\hline
\end{tabular}

Fonte: Adaptado de Cupertino e Brandstetter (2015)

\section{RESULTADOS E DISCUSSÕES}

Após verificação das ocorrências de falta de terminalidade, foram identificadas quatorze ocorrências para análise e estudo. Cada uma delas foram detalhadas no modelo apresentado nas Figuras 2 e 3.

Figura 2 - Registro fotográfico da ocorrência da sanca fechada não executada e da análise de causa raiz
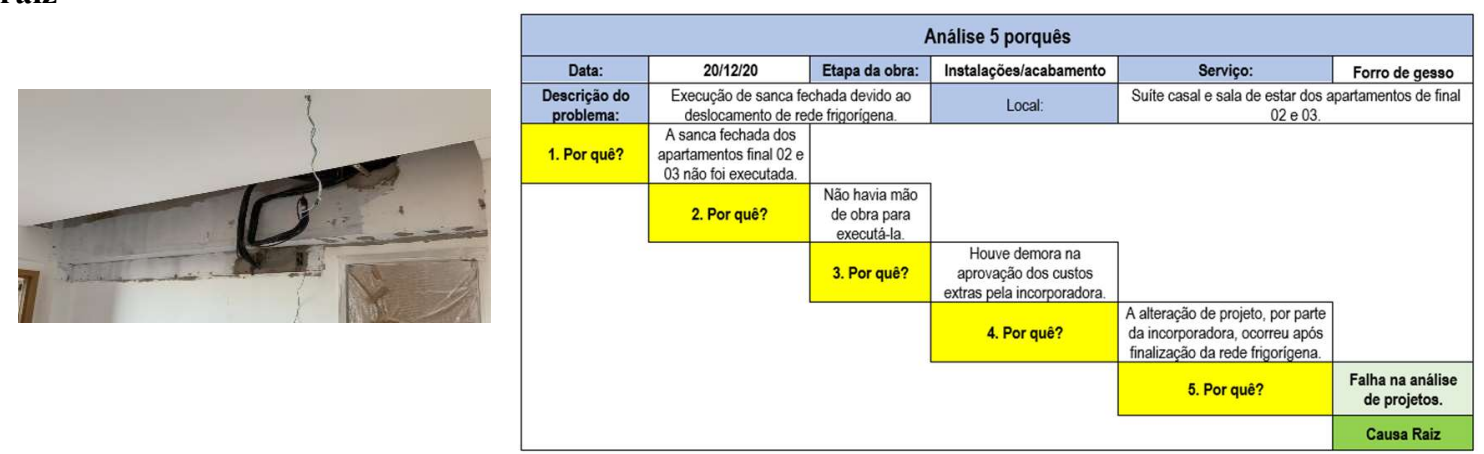

Fonte: Autoria própria (2021) 
Figura 3 - Registro fotográfico da ocorrência do revestimento não concluído acima da bancada e da análise de causa raiz
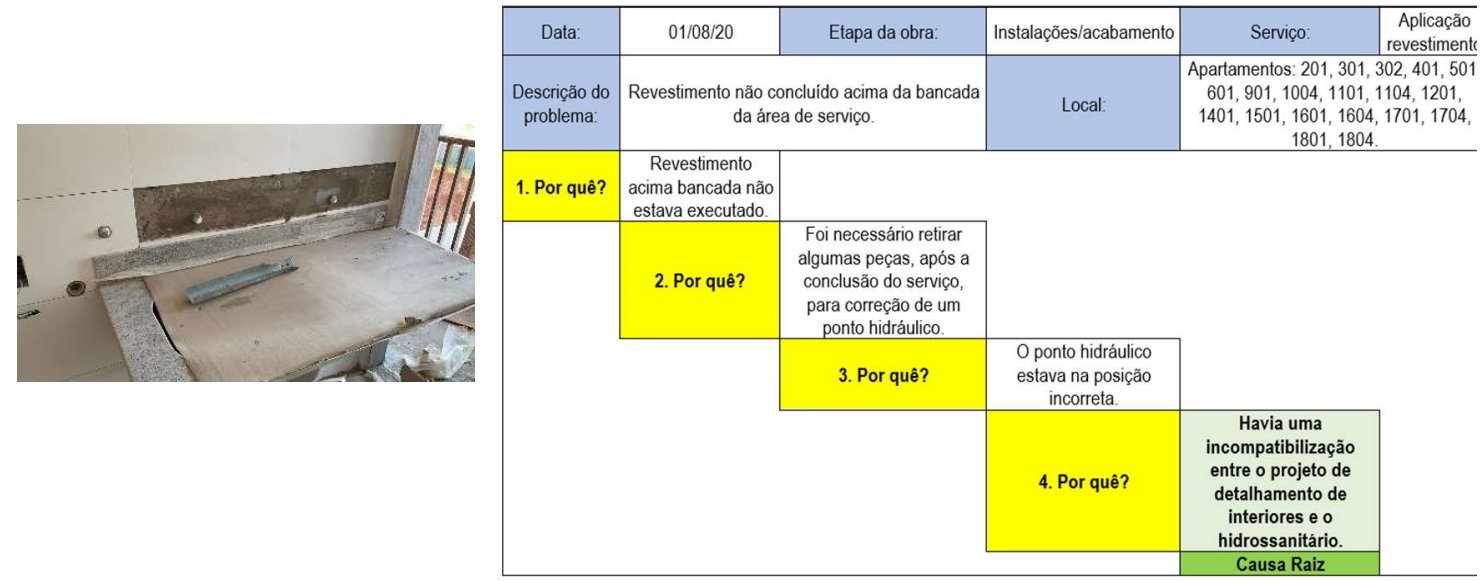

Fonte: Autoria própria (2021)

A Figura 4 ilustra o Diagrama de Pareto relativo à frequência das 14 ocorrências identificadas.

Figura 4 - Diagrama de Pareto da frequência das ocorrências
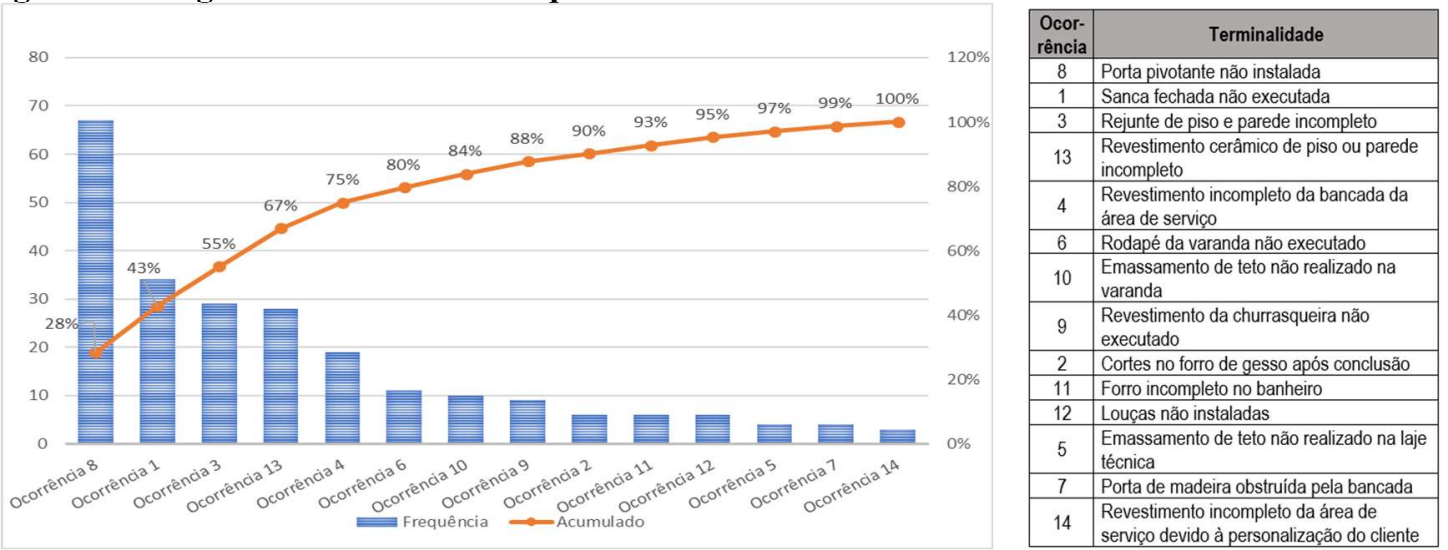

Fonte: Autoria própria (2021)

Foi possível analisar que quatro das ocorrências (28\%) são responsáveis por quase $70 \%$ do número total de faltas de terminalidade coletadas. Posteriormente foi realizada a análise da causa raiz, conforme apresentado na Figura 5.

Figura 5 - Gráfico da frequência de macrocategorias de causa raiz

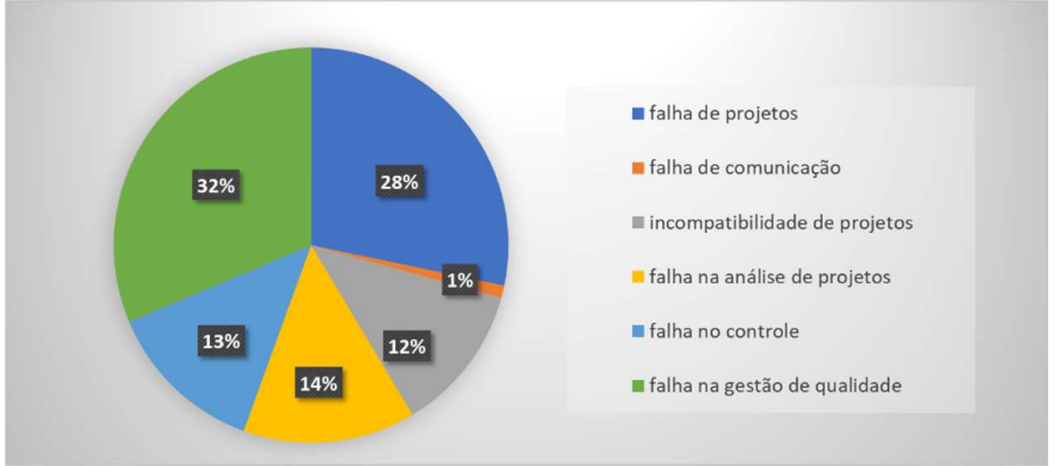

Fonte: Autoria própria (2021)

Todavia, essa análise quantitativa nem sempre expressa o que realmente mais impacta o custo da obra, o cronograma e a contratação de nova mão de obra, por exemplo. Partindo 
desse pressuposto, foi criado o índice de risco para cada ocorrência, calculado a partir do produto dos índices de impacto e frequência, apresentado na Tabela 1.

Tabela 1 - Tabela resumo do risco por ocorrência

\begin{tabular}{|c|c|c|c|c|c|}
\hline $\begin{array}{l}\text { Ocor- } \\
\text { rência }\end{array}$ & Terminalidade & Categoria & Impacto & $\begin{array}{c}\text { Frequên- } \\
\text { cia }\end{array}$ & $\begin{array}{l}\text { Índice } \\
\text { de risco }\end{array}$ \\
\hline 1 & Sanca fechada não executada & $\begin{array}{c}\text { Falha na análise de } \\
\text { projetos }\end{array}$ & 3 & 3 & 9 \\
\hline 2 & Cortes no gesso após conclusão & Falha no Controle & 1 & 1 & 1 \\
\hline 3 & Rejunte de piso e parede incompleto & $\begin{array}{c}\text { Falha na gestão de } \\
\text { qualidade }\end{array}$ & 2 & 3 & 6 \\
\hline 4 & $\begin{array}{l}\text { Revestimento incompleto da bancada da } \\
\text { área de serviço }\end{array}$ & $\begin{array}{l}\text { Incompatibilização } \\
\text { de projetos }\end{array}$ & 2 & 2 & 4 \\
\hline 5 & $\begin{array}{l}\text { Emassamento de teto não realizado na laje } \\
\text { técnica }\end{array}$ & $\begin{array}{l}\text { Falha na gestão de } \\
\text { qualidade }\end{array}$ & 1 & 1 & 1 \\
\hline 6 & Rodapé da varanda não executado & Falha no Controle & 2 & 2 & 4 \\
\hline 7 & Porta de madeira obstruída pela bancada & Falha no Controle & 1 & 1 & 1 \\
\hline 8 & Porta pivotante não instalada & Falha de projetos & 1 & 3 & 3 \\
\hline 9 & $\begin{array}{l}\text { Revestimento da churrasqueira não } \\
\text { executado }\end{array}$ & Falha no Controle & 2 & 2 & 4 \\
\hline 10 & $\begin{array}{l}\text { Emassamento de teto não realizado na } \\
\text { varanda }\end{array}$ & $\begin{array}{c}\text { Incompatibilização } \\
\text { de projetos }\end{array}$ & 1 & 2 & 2 \\
\hline 11 & Forro incompleto no banheiro & $\begin{array}{l}\text { Falha na gestão de } \\
\text { qualidade }\end{array}$ & 2 & 1 & 2 \\
\hline 12 & Louças não instaladas & $\begin{array}{l}\text { Falha na gestão de } \\
\text { qualidade }\end{array}$ & 2 & 1 & 2 \\
\hline 13 & $\begin{array}{l}\text { Revestimento cerâmico de piso ou parede } \\
\text { incompleto }\end{array}$ & $\begin{array}{c}\text { Falha na gestão de } \\
\text { qualidade }\end{array}$ & 3 & 3 & 9 \\
\hline 14 & $\begin{array}{l}\text { Revestimento incompleto da área de } \\
\text { serviço devido à personalização do cliente }\end{array}$ & $\begin{array}{l}\text { Falha de } \\
\text { comunicação }\end{array}$ & 2 & 1 & 2 \\
\hline
\end{tabular}

Fonte: Autoria própria (2021)

A Figura 6 ilustra a análise de risco em cada macrocategoria.

Figura 6 - Análise risco em cada macrocategoria

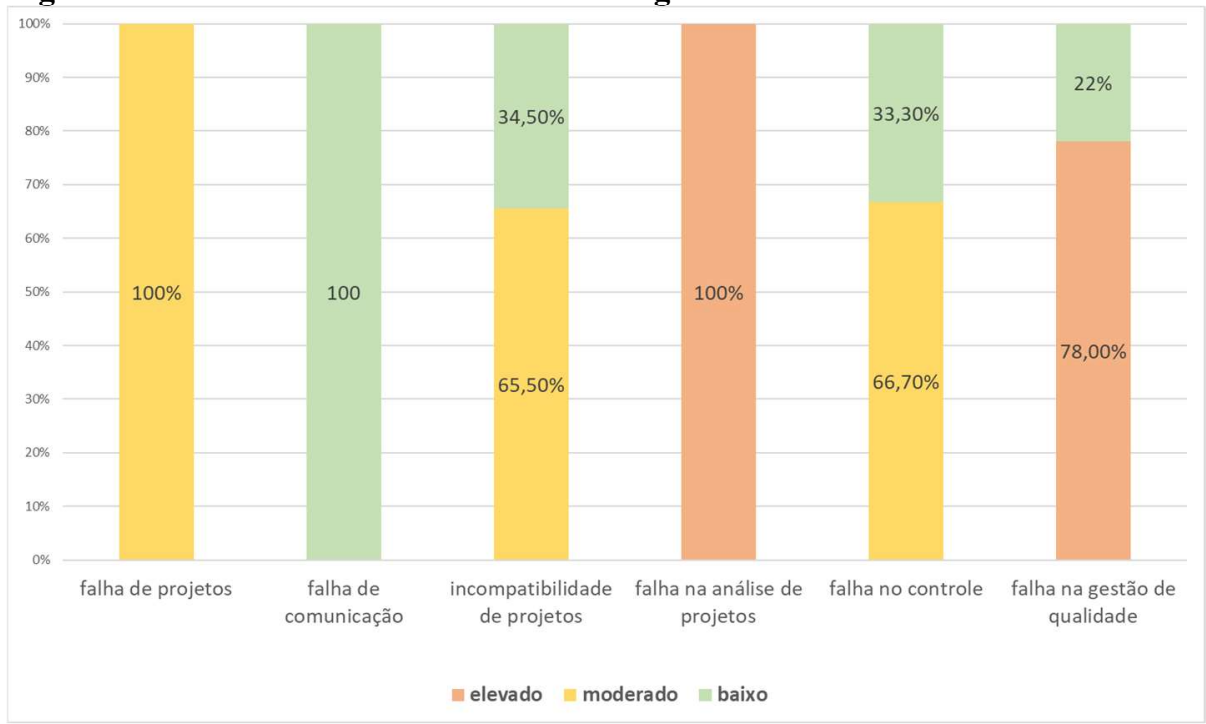

Fonte: Autoria própria (2021) 
As categorias de falha na análise de projetos e falha na gestão de qualidade apresentaram a maioria de suas ocorrências classificadas com risco elevado, o que implica em uma análise crítica das etapas que se relacionam com o serviço e controle, para que as ocorrências não se repitam em empreendimentos futuros.

\section{CONSIDERAÇÕES FINAIS}

Para qualidade, eficiência e, consequentemente, melhoria das construções é necessário que exista um planejamento bem definido que seja retroalimentado semanalmente com o intuito de identificar antecipadamente possíveis gargalos que podem impactar no cronograma da obra.

Desse modo o controle deve atuar de forma efetiva durante todas as etapas do processo, permitindo com que os gestores atuem diretamente nas causas dos problemas. Entre eles, a falta de terminalidade é um ponto que necessita de atenção especial pois ocasiona inúmeros danos em uma obra, entre eles, atrasos, custos extras, recontratação de mão de obra e complicações em serviços subsequentes.

No estudo de caso apresentado, entre os serviços estudados e as macrocategorias elencadas, pode-se concluir que alguns pontos necessitam de maior atenção em relação à solução das causas reais das faltas de terminalidade. Destacaram-se aquelas relacionadas à gestão da qualidade com cerca de $31 \%$ das causas raízes, como falhas relacionadas à não verificação do serviço, onde $78 \%$ estão associadas a um índice de risco elevado.

Ressalta-se que as falhas relacionadas ao projeto, seja por incompatibilizações, por análise ou solução técnica, somam $54 \%$ das causas associadas, sendo sua maioria associada a índices de risco moderado ou elevado.

O exemplo proposto pelo trabalho pode ser aplicado em diferentes tipologias de empreendimentos, auxiliando gestores quanto à priorização de ações para mitigação do risco associado aos serviços inacabados não planejados.

$\mathrm{O}$ entendimento do risco relacionado à falta de terminalidade de serviços permite $\mathrm{o}$ estabelecimento de medidas de controle e ações preventivas mais assertivas acerca do planejamento e controle em casos similares futuros.

\section{REFERÊNCIAS}

AKHUND, M. A.; IMAD, H. U.; MEMON, N. A.; SIDDIQUI, F.; KHOSO, A. R.; PANHWAR, A. A. Contributing factors of time overrun in public sector construction projects. Engineering, Technology \& Applied Science Research, v. 8, n. 5, p. 33693372, 2018.

ASSOCIAÇÃO BRASILEIRA DE NORMAS TÉCNICAS. NBR ISO 31000: gestão de risco: princípios e diretrizes. Rio de Janeiro, 2018.

ASSOCIAÇÃO BRASILEIRA DE NORMAS TÉCNICAS. NBR ISO/IEC 31010: gestão de risco: técnicas para o processo de avaliação de riscos. Rio de Janeiro, 2012.

BRANDSTETTER, M. C. G. O.; RIBEIRO, H. R. O. Causas de custos adicionais e impacto financeiro em obras públicas sob a perspectiva da gestão de risco. Ambiente Construído, Porto Alegre, v. 20, n. 1, p. 41-63, jan./mar. 2020.

CUPERTINO, D.; BRANDSTETTER, M. C. G. de O. Proposição de ferramenta de gestão pós-obra a partir dos registros de solicitação de assistência técnica. Ambiente Construído, Porto Alegre, v. 15, n. 4, p. 243-265, out./dez. 2015.

FIREMAN, M. C. T. et al. Integrating Production and Quality Control: monitoring making-do and unfinished work. In: ANNUAL CONFERENCE OF THE 
INTERNATIONAL GROUP FOR LEAN CONSTRUCTION, 21th, Fortaleza, 2013. Proceedings... Fortaleza, 2013.

FORMOSO, C. T. et al. An Exploratory Study on the Measurement and Analysis of Making-Do in Construction Sites. In: ANNUAL CONFERENCE OF THE INTERNATIONAL GROUP FOR LEAN CONSTRUCTION, 19th, Lima, 2011. Proceedings... Lima, 2011.

FORMOSO, C. T.; SOMMER, L.; KOSKELA, L.; ISATTO, E. L. The identification and analysis of making-do waste: insights from two Brazilian construction sites. Ambiente Construído, Porto Alegre, v. 17, n. 3, p. 183-197, jul./set. 2017.

MACHADO, R. L. A Sistematização de Antecipações Gerenciais no Planejamento da Produção de Sistemas da Construção Civil. Florianópolis, 2003. Tese (Doutorado em Engenharia de Produção) - Programa de Pós-Graduação em Engenharia de Produção, Universidade Federal de Santa Catarina, Florianópolis, 2003.

RENUKA, S. M.; KAMAL, S.; UMARANI, C. A model to estimate the time overrun risk in construction projects. Theoretical and empirical researches in urban management, v. 12, n. 2, p. 64-76, 2017.

SANTOS, D. de G. Modelo de Gestão de Processos na Construção Civil Para Identificação de Atividades Facilitadoras. Florianópolis, 2004, 219 f. Tese (Doutorado em Engenharia de Produção) - Programa de Pós-Graduação em Engenharia de Produção, Universidade Federal de Santa Catarina, Florianópolis, 2004.

SANTOS, P. R. R.; SANTOS, D. de G. Investigação de perdas devido ao trabalho inacabado e o seu impacto no tempo de ciclo dos processos construtivos. Ambiente Construído, Porto Alegre, v. 17, n. 2, p. 39-52, abr./jun. 2017.

SOMMER, L. Contribuições Para Um Método de Identificação de Perdas Por Improvisação em Canteiros de Obras. Porto Alegre, 2010. 150 f. Dissertação (Mestrado em Engenharia Civil) - Programa de Pós-Graduação em Engenharia Civil, Universidade Federal do Rio Grande do Sul, Porto Alegre, 2010.

ULHÔA, C. G. Método de apoio ao planejamento para minimização de atrasos em obras. 2017. 176 f. Dissertação (Mestrado em Engenharia Civil). Programa de PósGraduação em Geotecnia, Estruturas e Construção Civil, Universidade Federal de Goiás, Goiânia, 2017.

ULHÔA, C. G. Proposição de diretrizes focadas na gestão para melhorias no controle de prazo de construção de empreendimento - Estudo de caso em obras da região de Brasília-DF e Goiânia-GO. 2012. 221 f. Tese (Doutorado em Estruturas e Construção Civil). Universidade de Brasília, Brasília, 2012. 